\title{
Correlation of ECG Changes with Coronary Angiographic Findings in Patients of Coronary Artery Disease
}

\author{
Ayush P. Somani ${ }^{1}$, Satish R. Khadse ${ }^{2}$, Sunil Kumar ${ }^{3}$, Sourya Acharya ${ }^{4}$, Shreya C. Gattani ${ }^{5}$, Amit H. Baheti ${ }^{6}$ \\ ${ }^{1}$ Department of General Medicine, JNMC, DMIMS, Sawangi, Meghe, Wardha, Maharashtra, India. \\ ${ }^{2}$ Department of Cardiology, JNMC, DMIMS, Sawangi, Meghe, Wardha, Maharashtra, India. \\ ${ }^{3}$ Department of General Medicine, JNMC, DMIMS, Sawangi, Meghe, Wardha, Maharashtra, India. \\ ${ }^{4}$ Department of General Medicine, JNMC, DMIMS, Sawangi, Meghe, Wardha, Maharashtra, India. \\ ${ }^{5}$ Department of General Medicine, JNMC, DMIMS, Sawangi, Meghe, Wardha, Maharashtra, India. \\ ${ }^{6}$ Department of General Medicine, JNMC, DMIMS, Sawangi, Meghe, Wardha, Maharashtra, India.
}

\section{ABSTRACT}

\section{BACKGROUND}

Coronary Artery Disease (CAD) is the most common type of heart disease. Assessment of coronary artery disease (CAD) is the starting point of determination of prognosis of patients presenting with clinical symptoms. ECG reflects the physiology of the myocardium during ischemia, while Coronary Angiography identifies vessel anatomy. In the present study, we aimed to correlate ECG changes with coronary angiographic findings along with prediction of number of vessels involved in patients of coronary artery disease. This study also intended to determine sensitivity and specificity of ECG for diagnosis of coronary artery disease using CAG as gold standard.

\section{METHODS}

This cross-sectional study was carried out for a period of 2 years at Acharya Vinobha Bhave Rural Hospital, a tertiary rural health institute. 200 patients above 18 years of age who presented with or without symptoms and were willing for ECG and Angiography were included in study. Patients with valvular and congenital heart disease were excluded. Patients were divided into groups according to ECG changes and correlated with coronary angiography findings.

\section{RESULTS}

In the present study, the sensitivity and specificity of ECG to diagnose myocardial infarction or ischemia (AWMI / AWI, IWMI / IWI, and LWMI / LWI) ranged from $87.50 \%$ to $96.63 \%$ and $91.30 \%$ to $95.45 \%$, respectively. Diagnostic accuracy of ECG to detect myocardial infarction or ischemia (AWMI / AWI, IWMI / IWI, and LWMI / LWI) ranged from $89.36 \%$ to $96.40 \%$.

\section{CONCLUSIONS}

ECG is used as reliable tool for the diagnosis of coronary artery disease due to its high sensitivity and specificity, though CAG is the Gold Standard method for the diagnosis of CAD.

\section{KEY WORDS}

ECG, Coronary Angiography, Myocardial Infarction/Ischemia
Corresponding Author:

Ayush P. Somani,

Department of General Medicine,

JNMC, DMIMS, Sawangi, Meghe,

Wardha, Maharashtra, India.

E-mail:dr.ayush.somani@gmail.com

DOI: $10.14260 / \mathrm{jemds} / 2020 / 481$

How to Cite This Article:

Somani AP, Khadse SR, Kumar S, et al. Correlation of ECG changes angiographic findings in patients of coronary artery disease. J. Evolution Med. Dent. Sci. 2020;9(31):2212-2216, DOI: $10.14260 /$ jemds $/ 2020 / 481$

Submission 20-04-2020,

Peer Review 22-06-2020,

Acceptance 29-06-2020,

Published 03-08-2020.

Copyright (c) 2020 JEMDS. This is an open access article distributed under Creative Commons Attribution License [Attribution 4.0 International (CC BY 4.0)] 


\section{BACKGROUND}

Cardiovascular diseases (CVDs) are the most prevalent and grave disorders in industrialized nations and are increasing in developing nations as well. Globally, CVDs are now recognized as the leading cause of disease burden and deaths.[1-3]As per the WHO estimates (2016), CVDs resulted in death of around 17.9 million people, symbolizing approximately $31 \%$ of all worldwide deaths. ${ }^{[4]}$

Initial diagnosis of CAD is primarily dependent on approaches that involve some risk stratification of risk and includes medical history, physical examination, measurement of serum cardiac markers, stress testing, an electrocardiogram (ECG), and coronary arteriography (CAG). Thus, the diagnosis depends on the cautious analysis of ischemic cardiac manifestations. The diagnosis of CAD requires quick decisionmaking, as it is a life-threatening condition. ${ }^{[5]}$

Together with history taking and physical examination, comprehending the ECG findings is a basic element of the first evaluation of patients presenting with symptoms doubtful to be associated with ischemia of myocardium. The clinical usefulness of the ECG is determined through its prompt availability as an inexpensive, non-invasive, and highly flexible test. Apart from diagnosing conditions such as conduction disturbances, myocardial ischemia, and arrhythmias, ECG may also be used to diagnose disorders with increased sensitivity to sudden cardiac death (e.g. QT prolongation syndromes) or life-threatening metabolic disturbances (e.g. hyperkalaemia).[6,7]

In the past 3 decades, modern and more unambiguous imaging techniques have been introduced, namely echocardiography, cardiac computerized tomography (CT), nuclear scanning, and magnetic resonance imaging (MRI) and these provide additional information obtained from the ECG in evaluation of the heart in variety of stable conditions. However, as a result of its easy availability, cheap cost and quick interpretation, the ECG is still a major non-invasive cardiac imaging tool..[7] However, ECG and clinical symptoms have limited scope to provide information linked to the amount and location of coronary artery stenosis.[8]

Both coronary angiography (CAG) and heart catheterization are priceless techniques employed to detect and quantify CAD, identify valvular and other structural abnormalities, and measure the hemodynamic parameters. CAG is the gold standard technique used for identification of the area and degree of atherosclerotic CAD. The limitations observed with any invasive technique, procedure-related and specific patient-dependent complications, are inherent to the CAG.

For the diagnosis of CAD, some studies have used more cost-benefit, simple, and accurate tools such as the standard 12-lead ECG. Moreover, the diagnosis and management of patients with CAD has depended to a large extent on the ECG for evaluating changes in ST-segment, $\mathrm{T}$ wave, and $\mathrm{Q}$ wave associated with ischemia. As per the literature available in western world, the sensitivity and specificity of ECG, compared to that of CAG, is low to accurately detect the CAD. ${ }^{[9-12]}$ However, such studies from the perspective of adult Indian patients with CAD are lacking.

The use of non-invasive assessment tools, such as ECG, for forecasting $\mathrm{CAD}$ has been advised primarily because it provides patient convenience, safety, and faster performance.[13] Thus, the present study was performed to correlate the different ECG changes with CAG findings in patients of CAD.

\section{METHODS}

This cross-sectional study was accomplished in the MICU under Medicine department at a tertiary health care centre in central India from October 2017 to September 2019, after receiving Clearance DMIMS[DU]/IEC/2017-18/6758 from the institutional ethical committee.

Acute MI or ischemia was initially identified from history, physical examination, standard 12 lead ECG, and cardiac enzymes (Troponin I), 2 D Echo. Other history obtained from the patients included the age, sex, and risk factors (such as smoking, family history, dyslipidaemia, diabetes mellitus, and hypertension). Presenting symptoms such as chest pain, sweating, palpitation, and breathlessness were recorded. The study included patients aged $>18$ years and newly diagnosed cases of MI or myocardial ischemia. Patients presented with the symptoms of MI or ischemia and with or without ECG changes or patients without symptoms of MI or ischemia, but with ECG changes. Patient who were willing to undergo CAG for evaluation of CAD and had obstructive CAD involving left anterior descending artery (LAD), right coronary artery (RCA), or left circumflex artery (LCX) were included in the study. While, study excluded patients with known congenital heart disease, valvular heart disease, electrolyte imbalance, implanted pacemakers, or valve prostheses.

A standard 12-lead ECGs was recorded and analysed by a single experienced person, who was blinded to both the patient history and angiographic findings. According to the localization of MI or ischemia, patients were divided into four groups, namely - Groups I: Anterior wall myocardial infarction (AWMI) or Anterior wall ischemia (AWI); Group II: Inferior wall myocardial infarction (AWMI) or Inferior wall ischemia (AWI); Group III: Lateral wall myocardial infarction (AWMI) or Lateral wall ischemia (AWI); and Group IV: Normal ECG.

Finally, to identify the coronary artery with obstruction, coronary angiography (CAG) was performed. An investigator who was blinded to the ECG findings, assessed the CAG films. The coronary artery with infarction was identified from significant stenosis ( $>70 \%$ ) or total occlusion of the LAD, RCA or LCX. Based on the coronary angiographic findings, subjects were divided into single vessel disease (SVD), double vessel disease (DVD), triple vessel disease (TVD), and normal vessels.

\section{Sample Size Calculation}

Sample size was calculated on the basis of the sensitivity and the following formula was used:

$\frac{\mathrm{Z}_{1-\frac{\alpha}{2}}^{2} \mathrm{p}(1-\mathrm{p})}{\mathrm{d}^{2}}$

$\mathrm{p}=$ sensitivity of ECG for diagnosing CAD from previous study $=51.5 \%=0.515140$

$\mathrm{d}=$ desired margin of error $=0.07$ $\mathrm{a}=0.05$ ( 2 sided)

$\mathrm{Z} 0.025=1.96$ 
The sample size turned out to be 195.3. Thus, 200 patients were recruited in the study.

\section{Statistical Analysis}

Data was collected, collated, and graphics were designed by Microsoft Office Excel 2013. Mean and standard deviation (SD) are descriptive values for quantitative data, and frequency and range for qualitative data.

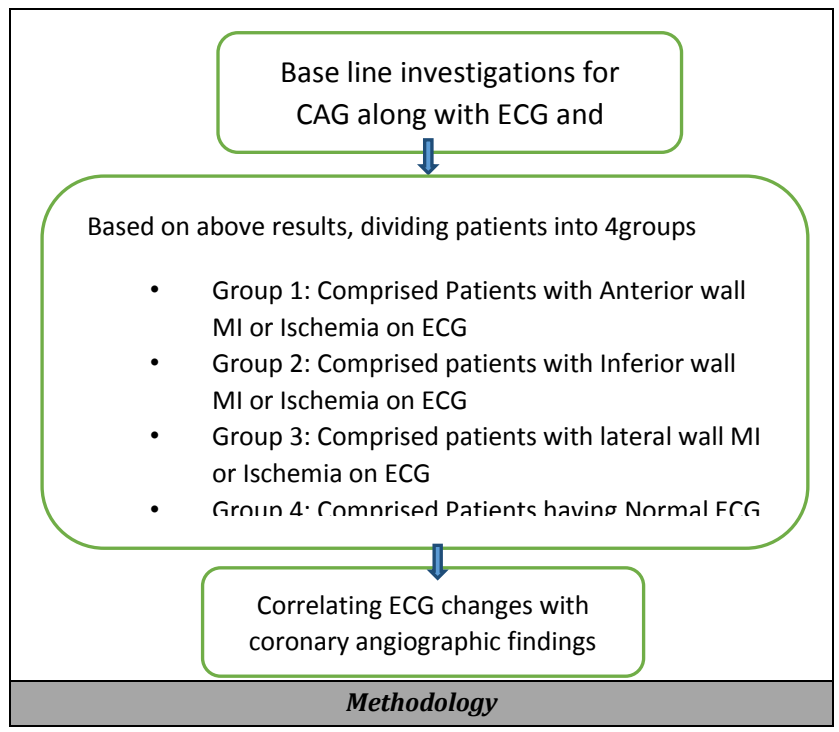

\section{RESULTS}

Table 2 shows that in majority of the study subjects, initial ECG taken at the time of presentation revealed an anterior wall MI or ischemia (43.5\%) followed by inferior wall MI or ischemia (33\%). As per the ECG findings, SVD was most common finding in AWMI/AWI (63.22\%) and IWMI/IWI (77.27\%). However, DVD was most common finding in LWMI/LWI (47.83\%).

Table 3 shows that LAD was predominantly involved in anterior wall MI or ischemia, RCA was predominantly involved in inferior wall MI or ischemia, while, LCx was predominantly involved in lateral wall MI or ischemia. Out of 110 patients of SVD, LAD involvement was observed in 52 (47.27\%) subjects, RCA involvement in $42(38.18 \%)$ subjects, and LCx involvement in $16(14.55 \%)$ subjects.

Table 4 shows out of 40 study subjects with double vessel disease, presented with following ECG changes, LAD + LCx was involved in 23 (57.5\%) subjects, LAD + RCA was involved in 10 $(25 \%)$ subjects, and RCA + LCx was involved in 7 (17.5\%) subjects.

Table 5 shows that out of 25 study subjects with triple vessel disease, presented with following ECG changes, 10 (40\%) had AWMI/AWI, 6 (24\%) had IWMI/IWI, and 6 (24\%) had LWMI/LWI.While, 3 (12\%) subjects had normal ECG findings.

Table 6 shows that the sensitivity, specificity, PPV, NPV, and diagnostic accuracy of ECG in diagnosing AWMI/AWI was 96.63\%, 95.45\%, 98.85\%, 87.50\%, and 96.40\%, respectively. The sensitivity, specificity, PPV, NPV, and diagnostic accuracy of ECG in diagnosing IWMI/IWI was 95.59\%, 95.45\%, 98.48\%, $87.50 \%$ and $95.56 \%$ respectively. The sensitivity, specificity, PPV, NPV, and diagnostic accuracy of ECG in diagnosing
LWMI/LWI was $87.50 \%, 91.30 \%, 91.30 \%, 87.50 \%$, and $89.36 \%$ respectively.

\begin{tabular}{|c|c|c|c|c|c|}
\hline & & & Num & $\operatorname{ver[200]}$ & Percentage \\
\hline \multicolumn{6}{|c|}{ Gender } \\
\hline \multicolumn{3}{|c|}{ Male } & \multicolumn{2}{|r|}{146} & 73 \\
\hline \multicolumn{3}{|c|}{ Female } & & 54 & 27 \\
\hline \multicolumn{6}{|c|}{ Age Group (Years) } \\
\hline \multicolumn{3}{|c|}{$<40$} & & 7 & 3.5 \\
\hline \multicolumn{3}{|c|}{$40-60$} & & 95 & 47.5 \\
\hline \multicolumn{3}{|c|}{$>60$} & & 98 & 48 \\
\hline \multicolumn{6}{|c|}{ Risk factors } \\
\hline \multicolumn{3}{|c|}{ Hypertension } & & 137 & 68.5 \\
\hline \multicolumn{3}{|c|}{ Smoking } & & 109 & 54.5 \\
\hline \multicolumn{3}{|c|}{ Diabetes mellitus } & & 95 & 47.5 \\
\hline \multicolumn{3}{|c|}{ Dyslipidaemia } & & 32 & 16 \\
\hline Family histor & $y$ of ischemic & leart disease & & 23 & 11.5 \\
\hline \multicolumn{6}{|c|}{ Table 1. Baseline Characteristics } \\
\hline \multirow{3}{*}{ ECG Findings } & \multicolumn{5}{|c|}{ Infarct Related Coronary Arteries } \\
\hline & SVD & DVD & TVD & Normal & Total \\
\hline & 55 & 21 & 10 & 1 & 87 \\
\hline †IWMI/IWI & 51 & 8 & 6 & 1 & 66 \\
\hline${ }^{{ }_{\text {LWMI}} / \mathrm{LWI}}$ & 4 & 11 & 6 & 2 & 23 \\
\hline Normal ECG & 0 & 0 & 3 & 21 & 24 \\
\hline Total (\%) & $110(56.5)$ & $40(23.5)$ & 25 (12.5) & $25(7.5)$ & $200(100)$ \\
\hline \multicolumn{6}{|c|}{$\begin{array}{l}\text { Table 2. Distribution of Subjects by Number of } \\
\text { Coronary Arteries Involvement }\end{array}$} \\
\hline \multicolumn{6}{|c|}{$\begin{array}{l}\text { *AWMI/AWI: Anterior wall Myocardial Infarction/ Anterior wall Ischemia } \\
\text { +IWMI/IWI: Inferior wall Myocardial Infarction/ Inferior wall Ischemia } \\
\text { }_{\text {LWWMI/LWI: Lateral wall Myocardial Infarction/ Lateral wall Ischemia }}\end{array}$} \\
\hline
\end{tabular}

\begin{tabular}{|cccc|}
\hline \multicolumn{4}{c|}{ Coronary Arteries Involved } \\
ECG Findings & LAD & RCA & LCx \\
AWMI/AWI & 52 & 0 & 3 \\
IWMI/IWI & 0 & 42 & 9 \\
LWMI/LWI & 0 & 0 & 4 \\
Normal ECG & 0 & 0 & 0 \\
Total & $\mathbf{5 2}(\mathbf{4 7 . 2 7 \% )}$ & $\mathbf{4 2}(\mathbf{3 8 . 1 8 \% )}$ & $\mathbf{1 6}(\mathbf{1 4 . 5 5 \% )})$ \\
Total & $\mathbf{1 1 0}$ \\
\hline \multicolumn{4}{|c|}{ Table 3. Single Vessel Disease - Coronary } \\
Angiography Correlation with ECG Changes \\
\hline
\end{tabular}

\begin{tabular}{|c|c|c|c|}
\hline \multirow{2}{*}{ ECG Findings } & \multicolumn{3}{|c|}{ Coronary Arteries Involved } \\
\hline & LAD + LCx & RCA + LCx & LAD + RCA \\
\hline AWMI/AWI & 14 & 2 & 5 \\
\hline IWMI/IWI & 5 & 2 & 1 \\
\hline LWMI/LWI & 4 & 3 & 4 \\
\hline Normal ECG & 0 & 0 & 0 \\
\hline Total & $23(57.5 \%)$ & $7(17.5 \%)$ & $10(25 \%)$ \\
\hline Total & \multicolumn{3}{|c|}{40} \\
\hline Table 4. Do & $\begin{array}{r}\text { ssel Disease } \\
\text { with } E\end{array}$ & $\begin{array}{l}\text { ary Angiogro } \\
\text { nges }\end{array}$ & elation \\
\hline
\end{tabular}

\begin{tabular}{|cc|}
\hline ECG Findings & $\begin{array}{c}\text { Coronary Arteries involved } \\
\text { LAD + RCA + LCx (\%) }\end{array}$ \\
AWMI/AWI & $10(40 \%)$ \\
IWMI/IWI & $6(24 \%)$ \\
LWMI/LWI & $6(24 \%)$ \\
Normal ECG & $3(12 \%)$ \\
Total & $\mathbf{2 5 ( 1 0 0 )}$ \\
\hline \multicolumn{2}{|c|}{ Table 5. Triple Vessel Disease - Coronary } \\
Angiography Correlation with ECG Changes \\
\hline
\end{tabular}

\begin{tabular}{|c|c|c|c|c|c|c|c|c|}
\hline 葛 & 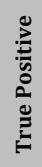 & 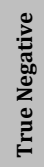 & 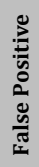 & 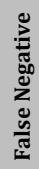 & 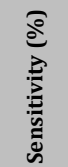 & 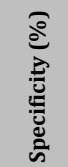 & 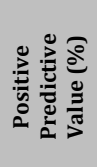 & 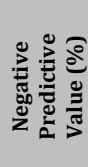 \\
\hline AWMI/AWI & 86 & 21 & 1 & 3 & 96.63 & 95.45 & 98.85 & 87.50 \\
\hline IWMI/IWI & 65 & 21 & 1 & 3 & 95.59 & 95.45 & 98.48 & 87.50 \\
\hline LWMI/LWI & 21 & 21 & 2 & 3 & 87.50 & 91.30 & 91.30 & 87.50 \\
\hline \multicolumn{9}{|c|}{ Table 6. Diagnostic Performance of ECG } \\
\hline
\end{tabular}

\section{DISCUSSION}

In India, Coronary artery disease is major cause of mortality due to cardiovascular disease. Moreover, due to its accessibility, recording an ECG is easy and cost effective. However, coronary angiography is costly and not easily available at every centre. In patients presenting with the 
symptoms of myocardial infarction or ischemia, correlating the ECG changes with CAG findings is really helpful. Thus, this present study was carried out in the Department of Medicine at a tertiary rural health care hospital, in central India over a period of 2 years i.e., from October 2017 to September 2019. During this period, we studied clinical presentation, risk factors, changes in ECG findings and CAG findings in 200 subjects of CAD. This was a cross sectional study performed after obtaining approval of the Institutional Ethics Committee (IEC) and signed informed consent of study subjects.

In the present study, majority of the study subjects, on ECG, had an anterior wall MI or ischemia (43.5\%) followed by inferior wall MI or ischemia (33\%) and Lateral wall MI/ ischemia (11.5\%). While, $12 \%$ subjects had no ECG changes. This changes were similar to those reported in other studies. Similar to the present study, Jankaret al. reported the anterior wall MI or ischemia (57\%) as the most common finding. Other finding on ECG were inferior wall MI or ischemia (19\%) followed by left ventricular hypertrophy (5\%) and left bundle branch block (3\%). Moreover, $16 \%$ of the patients had normal ECG findings.[13] In another study, Salunke et al. reported anterior wall MI (AWMI) (68\%) followed by IWMI (32\%) as the most common finding.[14]

We also observed, that majority of our study subjects, on CAG, had a SVD (55\%) followed by DVD (20\%), and TVD (12.5\%). While, only $12.5 \%$ of the study subjects had normal vessels. Overall, LAD (55\%) followed by RCA (42\%) and LCX artery (35.5\%) were the most commonly involved coronary arteries. A study conducted by Prajapati et al,[15] Swain et al,[16] Suresh et al ${ }^{[17]}$ reported findings similar to those in the present study while Jankar et al[13] reported DVD followed by a SVD and TVD as the most frequently observed CAG finding. The pattern of coronary artery involvement reported by Jankar et al.[13] Yusuf et al.[18] Badran et al[19] was similar to that observed in our study.

We concluded that LAD, RCA, and LCx were predominantly involved in anterior wall MI or ischemia, inferior wall MI or ischemia, and lateral wall MI or ischemia, respectively. In single vessel disease, LAD (47.27\%) followed by RCA (38.18\%) and LCx (14.55\%) were the most commonly affected coronary arteries. Swain et al.[20] and Tamrakar R et al.[21] observed findings similar to the present study.

Moreover, patients with triple vessel disease had MI or ischemia, in the following order: AWMI/AWI (40\%), IWMI/IWI (24\%), and LWMI/LWI (24\%). While, 3 (12\%) subjects had normal ECG findings. These findings were more or less similar to those found in study by Jankar et al.[13]

For routine assessment of more than $66 \%$ of the patients with angina, initial ECG forms an important routine diagnostic tool.[22] Previous studies assessed not only the cost-benefit and feasibility aspect of this tool, but also its predictive and prognostic values.[23] In the present study, the sensitivity and specificity to detect myocardial infarction or ischemia (AWMI/AWI, IWMI/IWI, and LWMI/LWI) ranged from $87.50 \%$ to $96.63 \%$ and $91.30 \%$ to $94.45 \%$. Diagnostic accuracy of ECG to detect myocardial infarction or ischemia (AWMI/AWI, IWMI/IWI and LWMI/LWI) ranged from 89.36\% to $96.40 \%$. Contrary to the findings of the present study, the previous studies by Fesmireet al.[24], Rude et al.[25], and Mahmoodzadeh et al.[26] showed relatively low sensitivity as well as partial low specificity of ECG for assessing the involvement of coronary arteries. Thus, the values of sensitivity and specificity of ECG observed in the present study were found to be conclusive and promising.

\section{CONCLUSIONS}

Anterior wall MI / ischemia followed by inferior wall MI / ischemia were the most common ECG findings. Single Vessel Disease (55\%) followed by Double Vessel Disease (20\%) were the most common CAG findings. LAD (55\%) followed by RCA (42\%) were the most common coronary arteries to be involved. LAD, RCA, and LCx artery were most commonly associated with AWMI, IWMI, and LWMI, respectively. In Double Vessel Disease, LAD + LCx (57.5\%) followed by LAD + RCA $(25 \%)$ and RCA + LCx (17.5\%) were the most common combinations of coronary arteries to be involved. Anterior wall MI / ischemia (40\%) was most commonly associated with triple vessel disease (LAD + RCA + LCx). ECG findings were normal in 24 subjects, while CAG was normal in 25 subjects. Sensitivity and specificity of ECG to detect CAD ranged from $87.50 \%$ to $96.63 \%$ and $91.30 \%$ to $95.45 \%$ respectively. The Diagnostic accuracy of ECG to detect CAD (AWMI / AWI, IWMI / IWI, and LWMI / LWI) ranged from $89.36 \%$ to $96.40 \%$.

The authors would like to acknowledge the contribution of all faculty members and junior resident doctors of Departement of Medicine, Acharya Vinobha Bhave Rural Hospital Sawangi [Meghe] Wardha along with the nursing and the paramedical staff of Medicine ICU and the Department of Cardiology.

Financial or Other Competing Interests: None.

\section{REFERENCES}

[1] GBD 2016 Causes of Death Collaborators. Global, regional, and national age-sex specific mortality for 264 causes of death, 1980-2016: a systematic analysis for the Global Burden of Disease Study 2016. Lancet 2017;390(10100):1151-210.

[2] GBD 2016 Mortality Collaborators. Global, regional, and national under-5 mortality, adult mortality, age-specific mortality, and life expectancy, 1970-2016: a systematic analysis for the Global Burden of Disease Study 2016. Lancet 2017;390(10100):1084-150.

[3] GBD 2016 Disease and Injury Incidence and Prevalence Collaborators. Global, regional, and national incidence, prevalence, and years lived with disability for 328 diseases and injuries for 195 countries, 1990-2016: a systematic analysis for the Global Burden of Disease Study 2016. Lancet 2017;390(10100):1211-59.

[4] World Health Organisation. Cardiovascular diseases (CVDs). 17th May 2017. https://www.who.int/en/newsroom/fact-sheets/detail/cardiovascular-diseases-(cvds)

[5] Antman EM, Loscalzo J. Ischemic heart disease. Chap- 293. In: Jameson JL, Fauci A, Kasper D, et al, eds. Harrison's principles of internal medicine. $19^{\text {th }}$ edn. New Delhi: McGraw-Hill Companies Inc 2015;1578-93.

[6] Goldberger AL. Electrocardiography. Chap- 293. In: Jameson JL, Fauci A, Kasper D, et al, eds. Harrison's 
principles of internal medicine. 19 $9^{\text {th }}$ edn New Delhi: McGraw-Hill Companies Inc 2015;1450-59.

[7] Birnbaum Y, Wilson JM, Fiol M, et al. ECG diagnosis and classification of acute coronary syndromes. Ann Noninvasive Electrocardiol 2014;19(1):4-14.

[8] Pontone G, Andreini D, Bartorelli AL, et al. A long-term prognostic value of CT angiography and exercise ECG in patients with suspected CAD. JACC Cardiovasc Imaging 2013;6(6):641-50.

[9] Horacek BM, Wagner GS. Electrocardiographic STsegment changes during acute myocardial ischemia. Card Electrophysiol Rev 2002;6(3):196-203.

[10] Fesmire FM, Percy RF, Bardoner JB, et al. Usefulness of automated serial 12-lead ECG monitoring during the initial emergency department evaluation of patients with chest pain. Ann Emerg Med 1998;31(1):3-11.

[11] Rude RE, Poole WK, Muller JE, et al. Electrocardiographic and clinical criteria for recognition of acute myocardial infarction based on analysis of 3,697 patients. Am J Cardiol 1983;52(8):936-42.

[12] Mahmoodzadeh S, Moazenzadeh M, Rashidinejad H, et al. Diagnostic performance of electrocardiography in the assessment of significant coronary artery disease and its anatomical size in comparison with coronary angiography. J Res Med Sci 2011;16(6):750-5.

[13] Jankar A, Edge Y, Palange P. A review of ECG changes and coronary angiographic findings in patient who underwent angiography. International Medical Journal 2016;3(5):450-8.

[14] Salunke KK, Khyalappa RJ. Role of electrocardiogram in identification of culprit vessel occlusion in acute ST elevation myocardial infarction in relation to coronary angiography. J Clin Prev Cardiol 2017;6(4):128-32.

[15] Prajapati J, Joshi H, Sahoo S, et al. AGE-related differences of novel atherosclerotic risk factors and angiographic profile among gujarati acute coronary syndrome patients. J Clin Diagn Res 2015;9(6):0C05-9.

[16] Swain L, Routray PN. Demographic and clinicangiographic profile of coronary artery disease in young adults: a retrospective observational study. Int J Res Med Sci 2018;6(7):2264-70.
[17] Suresh G, Subramanyam K, Kudva S, et al. Coronary artery disease in young adults: angiographic study- a singlecenter experience. Heart India 2016;4(4):132-5.

[18] Yusuf S, Hawken S, Ounpuu S, et al. Effect of potentially modifiable risk factors associated with myocardial infarction in 52 countries (the INTERHEART study): case-control study. Lancet 2004;364(9438):937-52.

[19] Badran HM, Elnoamany MF, Khalil TS, et al. Age-related alteration of risk profile, inflammatory response, and angiographic findings in patients with acute coronary syndrome. Clin Med Cardiol 2009;3:15-28.

[20] Mamun KSA, Awal A, Murshed AKMM. Correlation of ECG changes with coronary angiographic findings in acute inferior myocardial infarction. Medicine Today 2017;29(2):33-7.

[21] Tamrakar R, Bhatt YD, Kansakar S, et al. Acute myocardial infarction in young adults: study of risk factors, angiographic features and clinical outcome. Nepalese Heart Journal 2014;10(1):12-6.

[22] Daly CA, Clemens F, Sendon JL, et al. The clinical characteristics and investigations planned in patients with stable angina presenting to cardiologists in Europe: from the Euro Heart Survey of Stable Angina. Eur Heart J 2005;26(10):996-1010.

[23] Gibbons RJ, Balady GJ, Beasley JW, et al. ACC/AHA Guidelines for Exercise Testing. A report of the American College of Cardiology/American Heart Association Task Force on Practice Guidelines (Committee on Exercise Testing). J Am Coll Cardiol 1997;30(1):260-311.

[24] Fesmire FM, Percy RF, Bardoner JB, et al. Usefulness of automated serial 12-lead ECG monitoring during the initial emergency department evaluation of patients with chest pain. Ann Emerg Med 1998;31(1):3-11.

[25] Rude RE, Poole WK, Muller JE, et al. Electrocardiographic and clinical criteria for recognition of acute myocardial infarction based on analysis of 3,697 patients. Am J Cardiol 1983;52(8):936-42.

[26] Mahmoodzadeh S, Moazenzadeh M, Rashidinejad H, et al. Diagnostic performance of electrocardiography in the assessment of significant coronary artery disease and its anatomical size in comparison with coronary angiography. J Res Med Sci 2011;16(6):750-5. 